BARI-TH/280-97

\title{
Upward-going muons and neutrino oscillations
}

\author{
G. L. Fogli, E. Lisi, and A. Marrone \\ Dipartimento di Fisica and Sezione INFN di Bari, \\ Via Amendola 173, I-70126 Bari, Italy
}

\begin{abstract}
The available upward-going muon data from the Kamiokande, Baksan, MACRO, IMB, and SuperKamiokande experiments are reviewed and combined. Bounds on the neutrino mass and mixing parameters are derived for oscillations in two and three flavors. These bounds are not in significant conflict with the oscillation solution to the atmospheric neutrino flavor anomaly observed in the sub-GeV and multi-GeV energy range. The combination of all the available atmospheric data tends to favor the $\nu_{\mu} \leftrightarrow \nu_{e}$ channel with respect to the $\nu_{\mu} \leftrightarrow \nu_{\tau}$ channel, and to disfavor the threefold maximal mixing scenario.
\end{abstract}

PACS number(s): 96.40.Tv, 14.60.Ef, 25.30.Pt, 14.60.Pq 


\section{INTRODUCTION}

The observation of upward-going muons in underground detectors has long since been considered [1] as an effective tool to study the neutrino component expected in atmospheric showers 2 24]. Early anomalies in the muon event samples detected by the first two dedicated experiments [5,6] soon sparked investigations of possible neutrino oscillation effects [7,8].

The upward-going muon total rates measured in recent underground experiments appear to be approximately in agreement with the expectations, thus disfavoring large neutrino oscillation effects. This contrasts with the observed anomaly in the electron and muon contents of (semi)contained events induced by atmospheric neutrinos, that might signal flavor oscillation effects (see [9] for a recent review). The conflict between these two data sets is usually resolved by appealing to the large, noncancelable uncertainties affecting the calculation of the absolute muon rates [10 12]. In terms of neutrino oscillations, this implies that there are values of the oscillation parameters that explain the "anomalous" muon-toelectron flavor ratio without really conflicting with the "regular" upward muon data [13]15]. In this work we confirm and elucidate such point of view by performing an updated analysis of the upward-going muon data from five experiments: Kamiokande [16 19], Baksan [20 22], MACRO [23,24], IMB [25,26], and SuperKamiokande [27,28].

The paper is structured as follows. In Sec. II the available data on upward-going muons are reviewed, compared with each other and with the theoretical expectations (in the absence of oscillations), and then combined. In Sec. III the combined data on the muon flux angular distribution are used to derive bounds on the two-flavor and three-flavor neutrino oscillation parameters. These bounds are compatible with the neutrino oscillation solution to the atmospheric neutrino anomaly. It is shown that the inclusion of upward-going muon data in fits tends to favor the $\nu_{\mu} \leftrightarrow \nu_{e}$ oscillation channel and to disfavor the threefold maximal

\footnotetext{
${ }^{1}$ We add that the experimental systematics might also be more significant than usually assumed, see Sec. II B.
} 
mixing scenario. In Sec. IV we draw the conclusions of our work. This study is part of a vast research program aiming to analyze the world neutrino oscillation data, including the results of solar [29], atmospheric, [29 31] and laboratory (accelerator and reactor) 29, 32] neutrino experiments.

\section{EXPERIMENTAL DATA AND STANDARD EXPECTATIONS}

The experimental data on upward-going muons are usually given as distributions of either muon events or muon fluxes as a function of the zenith angle $\theta$, with $\cos \theta=0$ $(\cos \theta=-1)$ corresponding to horizontal (vertical) muons. The distribution of muon events depends upon technical specifications of the detector such as the efficiency and the geometric acceptance, that are often unpublished. The distribution of muon fluxes (when available) is instead deconvoluted for these effects, and thus allows a more direct comparison with the theoretical expectations. We describe in Sec. II A the more recent available data (either muon events or muon fluxes) and in Sec. II B the specific combination of data used in our oscillation analysis.

\section{A. Data from different experiments}

In the last few years, data on upward going muons have been collected by five experiments: Kamiokande [16 19], Baksan [20 22], MACRO [23,24], IMB [25,26], and SuperKamiokande 27,28]. The IMB experiment (completed) was performed in three distinct stages (phases 1, 2, and 3); we will comment both on the global results (IMB-1,2,3) [26] and on the results from the first two phases (IMB-1,2) [25]. Data from the pioneering NUSEX experiment [33 (eight events only) will not be considered. Since some experimental results have been reported only in conference proceedings, we compile the most recent data on upward-going muons for the benefit of the reader. The compilation is reported in Table \& and in Fig. 1, that we comment in parallel. 
Table @ reports, for each experiment, the relevant detector characteristics and the experimental muon data, compared with the corresponding expectations in ten angular bins. The theoretical muon fluxes refer to our calculation? with the following inputs: Bartol neutrino fluxes [36] (neglecting small, site-dependent geomagnetic effects), version "G" of the Martin-Stirling-Roberts $[\mathrm{MRS}(\mathrm{G})]$ structure functions [37] (i.e., the default choice of the CERN Parton Density Function Library [38,39]), and Lohmann-Kopp-Voss (LKV) muon energy losses in standard rock [40]. The use of the same input for the calculation of the expected muon fluxes will enable us to perform a uniform comparison with all the experiments.3 Notice that, for fixed inputs, the theoretical muon spectrum depends only on the energy threshold; therefore, the Baksan and MACRO experiments (both with $E_{\mu}>1 \mathrm{GeV}$ ) share the same expectations. All other experiments have higher thresholdst and correspondingly lower muon fluxes. Concerning the experimental data, we quote only the statistical errors, since the systematic errors in each bin are usually unpublished. The only notable exception is the MACRO analysis [23,24], where the systematic uncertainties are estimated to range from a few percent near the vertical to about $20 \%$ near the horizontal direction. We also report (last row in Table $\mathbb{I}$ ) the total muon flux, defined as the sum of the individual fluxes in each bin times the bin width, with (statistical) errors added in quadrature.

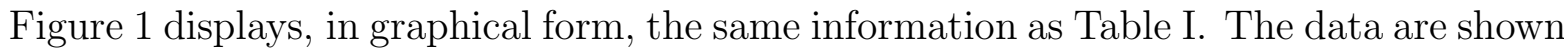
as dots with (statistical) error bars. Our calculations (in the absence of oscillations) are represented by the solid histograms. In addition, we report as dashed histograms the original

${ }^{2}$ A general description of the theoretical estimate of muon fluxes can be found, e.g., in [34, 35].

${ }^{3}$ It should be stressed, however, that alternative input choices for the neutrino fluxes or for the structure functions may induce variations as large as $\sim 30 \%$ in the normalization of the expected muon fluxes. This uncertainty will be included in the fits.

${ }^{4}$ In particular, the IMB threshold corresponds to $\sim 1.8 \mathrm{GeV}$ for phases 1 and 2 , to $\sim 1 \mathrm{GeV}$ for phase 3 , and to an effective value $\sim 1.4 \mathrm{GeV}$ for the total data sample (IMB-1,2,3). 
MonteCarlo simulations of the various collaborations. The ingredients of these simulations were: (1) Kamiokande [16, 18]: Volkova neutrino fluxes [41], Eichten-Hinchliffe-Lane-Quigg (EHLQ) structure functions [42,38, and Lohmann-Kopp-Voss (LKV) muon energy losses [40]; (2) Baksan [21,22]: Bartol neutrino fluxes [36], Morfin-Tung (MT) structure functions [43], and LKV muon energy losses; (3) MACRO [24]: Bartol neutrino fluxes, MT structure functions, and LKV muon energy losses; (4) IMB 25,26]: Volkova neutrino fluxes, EHLQ structure functions, and Bezrukov-Bugaev muon energy losses [44; (5) SuperKamiokande 28]: Bartol neutrino fluxes, EHLQ structure functions, and LKV muon energy losses. The differences between our calculations and the MonteCarlo simulations are relatively small.

The last panel in Fig. 1 shows the total number of muons that stopped in, or passed through, the IMB detector (phases 1, 2, and 3 combined). Unfortunately, it is difficult to calculate reliably the corresponding theoretical rates without detailed information about the IMB detector. The very definition of "passing" or "stopping" muon depends on unreproducible geometric cuts applied on an event-by-event basis. Their approximation with (more manageable) energy cuts (see, e.g., [14]) may bias the classification of events. Moreover, the absolute estimates of muon yields requires also the accurate knowledge of the detector geometrical acceptance and of its energy-dependent efficiency, which are not published. Therefore, we are not surprised that, just for IMB-1,2,3 (last panel of Fig. 1), our tentative calculation of the "passing" muon yield is not in good agreement with the published IMB MonteCarlo simulation [26]. For these reasons, we have not used the IMB-1,2,3 stopping and passing muon data in our analysis. We have instead used the muon flux distribution (published for IMB-1,2 [25]), that can be calculated more reliably and can also be compared with similar distributions from other experiments.

\footnotetext{
5 The muon flux distribution for IMB-1,2,3 is, unfortunately, unpublished.
} 


\section{B. Combination of data}

As Fig. 11 shows, the angular distributions measured by Kamiokande, Baksan, MACRO, IMB-1,2, and SuperKamiokande are roughly in agreement with the theoretical expectations. However, the data also show some "bumps" or "dips" that seem too large to be statistical fluctuations but also too random to suggest new physics - they are likely to be the effect of unknown experimental systematics. Let us consider, e.g., the MACRO and Baksan experimental spectra, that, as observed in the previous subsection, are characterized by the same threshold $\left(E_{\mu}>1 \mathrm{GeV}\right)$ and thus can be directly compared by superposition. Both the Kolmogorov-Smirnov and the $\chi^{2}$ tests give a $\ll 1 \%$ probability that these two distributions are the same within the statistical errors. This suggests that the experimental systematics might be nonnegligible (in some bins, at least), as indeed confirmed by the MACRO error analysis [24]. Unfortunately, no analogous error analysis has been reported by the Baksan Collaboration.

The presence of relatively large "dips and bumps" in the individual experimental spectra of Fig. 1 poses some practical problems in fitting the data with theoretical curves (that, conversely, are rather smooth even in the presence of neutrino oscillations). In fact, the oscillation fits to the individual spectra become dominated by the bins that exhibit the largest fluctuations, thus producing rather unstable and unreliable results. Therefore, it seems more reasonable to combine first the data from different experiments, in order to "average out" their fluctuations, and then to fit the neutrino oscillation parameters.

We have combined the five muon flux spectra of Fig. 1 into a single spectrum as follows. First, the experimental distributions have been "rescaled" to a common $1 \mathrm{GeV}$ threshold, by adding in each bin the theoretical contribution due to the change in threshold (no correction is necessary for MACRO and Baksan). The results are shown in Fig. 2(a), together with the theoretical expectation (solid histogram), now common to all experiments. Then

\footnotetext{
${ }^{6}$ Notice that the spread of the experimental data points in Fig. 2(a) is significant (a factor of about
} 
the data have been combined in each bin, using only the known, statistical errors. The resulting, combined distribution is shown in Fig. 2(b) (dots with small error bars): it appears smoother than each of the individual parent distributions, and thus more appropriate for fits. A delicate point is the estimate of the (nonnegligible but unknown) systematic errors.

The Particle Data Group recipe for scaling data errors as $\sqrt{\chi^{2} /\left(N_{\mathrm{DF}}-1\right)}$ [45] would give scaling factors between 1 and 2 in the various bins. Similarly, the addition of plausible, $\mathcal{O}(10 \%)$ systematic errors would give total errors $\sim 1.5$ times larger than the statistical errors. Guided by these estimates, we have chosen to rescale the statistical errors by a uniform factor 1.5 [large error bars in Fig. 2(b)] in order to obtain the "total" uncertainties. These "inflated" experimental errors have been used in all the following fits.

We stress that any conceivable combination of the present muon data necessarily involves some approximations or subjective choices, both because spectra with different thresholds cannot be compared directly and because no experiment (excepted MACRO) reports the systematic uncertainties in each bin. For instance, the (somewhat arbitrary) choice of a common threshold for rescaling the experimental spectra has some influence on the sensitivity of the fits to low values of the neutrino mass differences. Therefore, great care should be taken in interpreting the results of any fit to the available muon data, including those presented in the following Section. Hopefully, in the next few years the SuperKamiokande experiment will collect much higher muon statistics and will carefully estimate the systematic uncertainties of the flux distribution, so as to render such drawbacks gradually less significant in future analyses.

two in the muon fluxes), suggesting again and independently that large and not well-understood systematics might affect (at least some of) the experimental results. 


\section{UPWARD-GOING MUONS AND NEUTRINO OSCILLATIONS}

In this section we report the results of our two-flavor and three-flavor oscillation analysis of the upward muon spectrum shown in Fig. 2(b). Since this combined spectrum is in reasonable agreement with the expectations in the absence of oscillations, exclusion zones can be drawn in the neutrino oscillation parameter space. This contrasts with the flavor anomaly observed in contained and semicontained atmospheric neutrino events, which suggests nonzero neutrino masses and mixings [31]. However, as we will see, positive and negative indications are compatible, within the experimental and theoretical uncertainties, in some zones of the parameter space where the neutrino mixing is "intermediate" (i.e., neither zero nor maximal).

We use the three-flavor oscillation formalism defined in our previous works [29,31,32]. The first two of the three neutrino mass eigenstates $\left(\nu_{1}, \nu_{2}, \nu_{3}\right)$ are assumed to be essentially degenerate $\left(m_{1} \simeq m_{2}\right)$, and separated from the third state $\nu_{3}$ by a square mass gap $m^{2}=$ $\left|m_{3}^{2}-m_{1,2}^{2}\right|$. The flavor content of $\nu_{3}$ is parametrized as

$$
\nu_{3}=\sin \phi \nu_{e}+\cos \phi\left(\sin \psi \nu_{\mu}+\cos \psi \nu_{\tau}\right)
$$

where the mixing angles $\phi$ and $\psi$ range between 0 and $\pi / 2$. The cases $\phi=0, \psi=\pi / 2$, and $\psi=0$ correspond to pure two-flavor oscillations in the channels $\nu_{\mu} \leftrightarrow \nu_{\tau}, \nu_{e} \leftrightarrow \nu_{\mu}$, and $\nu_{e} \leftrightarrow \nu_{\tau}$, respectively (the latter case $\nu_{e} \leftrightarrow \nu_{\tau}$ is uninteresting for atmospheric neutrinos and will not be specifically considered). As shown in [31,32], it is useful to chart the parameter space through the logarithmic coordinates $\left(m^{2}, \tan ^{2} \phi, \tan ^{2} \psi\right)$. Our results will be presented in representative sections of this space.

For each value of the neutrino mass and mixing parameters, we calculate the theoretical muon flux spectrum with the inputs described in the caption to Table $\mathbb{E}$, including the matter oscillation effect for neutrino paths in the Earth 29,31. Then we evaluate the goodness-of-fit to the experimental distribution in Fig. 2(b) through a $\chi^{2}$ test. The $\chi^{2}$ quadratic form includes both the uncorrelated total experimental errors and the correlated 
theoretical uncertainties of the absolute neutrino fluxes, cross sections, and muon energy losses in the rock. The $1 \sigma$ total theoretical error is conservatively assumed to be $30 \%$, with a bin-by-bin correlation coefficient equal to +1 . For both two-flavor and three-flavor oscillations the minimum value of $\chi^{2}$ is searched, and C.L. contours are drawn at the values of $\Delta \chi^{2}=\chi^{2}-\chi_{\min }^{2}$ appropriate to $N_{\mathrm{DF}}=2$ and $N_{\mathrm{DF}}=3$, respectively (see also [31]). We will combine in different ways the following data sets, in order to show their relative "weight" in driving the fit: upward-going muons (U) from Kamiokande, IMB-1,2, Baksan, MACRO, and SuperKamiokande; sub-GeV (S) $\mu$-like and $e$-like events from Kamiokande, IMB, NUSEX, and Frejus [31]; multi-GeV (M) $\mu$-like and $e$-like events from Kamiokande [46], both binned $(\mathrm{Mb})$ and unbinned $(\mathrm{Mu})$ [31]. The data sets $\mathrm{S}, \mathrm{Mu}$, and $\mathrm{Mb}$ are analyzed as in [31].

\section{A. Two-flavor oscillations}

Figure 3 shows the results of our analysis of $\nu_{\mu} \leftrightarrow \nu_{\tau}$ oscillations. In the first panel (U) only the upward-going muon data are fitted. The symmetry of the C.L. contours with respect to the vertical axis $\phi=\pi / 4$ reflects the absence of matter effects in the $\nu_{\mu} \leftrightarrow \nu_{\tau}$ channel [31]. As expected, large neutrino mixing is excluded (for $m^{2} \gtrsim 10^{-3}$ ). This negative indication, however, does not rule out the evidence for oscillations coming from sub-GeV and multi-GeV binned or unbinned data ( $\mathrm{S}+\mathrm{Mb}$ and $\mathrm{S}+\mathrm{Mu}$ panels). In fact, the combination of all atmospheric data still favors the oscillation hypothesis, as evidenced in the plots labeled "S+Mb+U" (sub-GeV + multi-GeV binned + up-going $\mu$ ) and "S+Mu+U" (sub-GeV + multi-GeV unbinned + up-going $\mu$ ).

Notice that the addition of the upward-going muon data reinforces the exclusion of large $\nu_{\mu} \leftrightarrow \nu_{\tau}$ mixing (required by the negative results of the NUSEX and Frejus experiments [31]), but does not alter dramatically the oscillation fit to sub- $\mathrm{GeV}$ and multi-GeV atmospheric neutrino data. In particular, for large $m^{2}$ the "S+Mu+U" bounds on $\tan ^{2} \phi$ are only slightly narrower than those without upward $\mu$ data ("S+Mu" panel). This observation is relevant 
for those phenomenological models in which the controversial zenithal anomaly of multi$\mathrm{GeV}$ data is discarded, such as the "minimum sacrifice" scenario studied in 47,48. We have verified that the solution to the neutrino oscillation evidence proposed in such scenario survives after the inclusion of the upward-going muon data, although with a slightly worse $\chi^{2}$

Figure 4 shows the results of our analysis of $\nu_{e} \leftrightarrow \nu_{\mu}$ oscillations. The panels are labeled as in Fig. 3. Notice that the bounds from upward-going muon data in the $\nu_{e} \leftrightarrow \nu_{\mu}$ channel are considerably weaker than in the $\nu_{\mu} \leftrightarrow \nu_{\tau}$ channel, and basically exclude a relatively small zone at $90 \%$ C.L. for large mixing and $m^{2} \simeq 0.06-0.3 \mathrm{eV}^{2}$ (no constraints appear at 99\% C.L.). In fact, in the $\nu_{e} \leftrightarrow \nu_{\mu}$ channel the $\nu_{\mu}$ disappearance is partially compensated by the conversion of $\nu_{e}$ (initially present in the beam) into $\nu_{\mu}$. The "suppression" of the muon disappearance is also influenced by matter effects, that are responsible of the leftright asymmetry in the plots (see [31]). Also in this case, the bounds from sub-GeV and multi-GeV data ("S+Mu" and "S+Mb" panels) are only slightly altered by the inclusion of upward-going muon data ("S+Mu+U" and "S+Mb+U" panels, respectively).

In conclusion, the upward-going muon data tend to disfavor large mixing in the $\nu_{\mu} \leftrightarrow \nu_{\tau}$ channel and, more weakly, in the $\nu_{\mu} \leftrightarrow \nu_{e}$ channel. This indication reinforces the exclusion of large mixing required by the negative sub-GeV results from NUSEX and Frejus. However, the indications for "intermediate" mixing derived by a global fit to the atmospheric data are not dramatically altered by the inclusion of the upward-going muon data. In particular, the $\nu_{\mu} \leftrightarrow \nu_{\tau}$ oscillation mode is still compatible with the "minimum sacrifice scenario" of Refs. [47,48], which is thus able to accommodate all present neutrino oscillation data, with the only exception of the non-flat zenith angle distribution measured in the Kamiokande multi-GeV experiment 46]. 


\section{B. Three-flavor oscillations}

Figure 5 reports the results of our analysis of upward-going muon data in the three-

flavor space $\left(m^{2}, \tan ^{2} \phi, \tan ^{2} \psi\right)$, shown as planar sections at representative values of $m^{2}$. We recall that, in each $\left(\tan ^{2} \psi, \tan ^{2} \phi\right)$ panel, the right side corresponds (asymptotically) to pure $\nu_{\mu} \leftrightarrow \nu_{e}$ oscillations, while the lower side correspond (asymptotically) to pure $\nu_{\mu} \leftrightarrow \nu_{\tau}$ oscillations. The excluded regions in Fig. 5 connect continuously these two subcases and show that also genuine three-flavor cases are disfavored by the available upward-going muon data. These cases correspond to large neutrino mixings. In particular, the threefold maximal mixing scenario 49,50, obtained for $\left(\sin ^{2} \psi, \sin ^{2} \phi\right)=(1,1 / 2)$, is rather strongly disfavored by the upward-going muon data.

The exact shapes of the excluded regions should be taken with some caution, since we have checked that they are rather sensitive to variations in the data and in their statistical treatment; therefore, new and more precise upward-going muon data from the SuperKamiokande experiment might alter significantly the bounds shown in Fig. 5. However, the following qualitative features seem to be relatively stable: 1) weaker bounds in the $\nu_{\mu} \leftrightarrow \nu_{e}$ channel; and 2) exclusion of large neutrino mixing and, in particular, of three-fold maximal mixing.

\section{Combination of all atmospheric data}

Figure 6 shows the results of a combined analysis of all the available sub-GeV, multi-GeV (binned), and upward-going muon data. As for Fig. 5 we recall that, because of the quality of the available upward-going muon data, these fits might change significantly with new, more accurate data. Nevertheless, some interesting, qualitative features are seen to emerge: 1) in general, $\nu_{e} \leftrightarrow \nu_{\mu}$ oscillations tend to give a better fit than $\nu_{\mu} \leftrightarrow \nu_{\tau}$ oscillations, as a result of the combined preference of multi-GeV data [31] and of weaker bounds from upward-going muon data for $\nu_{e} \leftrightarrow \nu_{\mu}$ transitions; and 2) the overall oscillation fit is reasonably good even 
for $m^{2}$ as small as $\sim 5 \times 10^{-4} \mathrm{eV}^{2}$. These indications suggest that oscillation signals might be seen better in long-baseline reactor experiments, that can probe the $\nu_{e}$ disappearance mode down to $m^{2} \sim 10^{-3} \mathrm{eV}^{2}$. If no oscillation signal is found in such experiments, large horizontal stripes will be excluded in the panels of Figs. 5 and 6 [32].

In conclusion, both the positive and the negative indications for flavor oscillations coming from all the available atmospheric neutrino data (including upward-going muons) can be made compatible, within the uncertainties, at intermediate values of the neutrino mixing angles. In fact, although the positive indications for $\nu$ oscillations favor large mixing, the negative results from upward-going muon experiments (as well as from some sub-GeV experiments like Frejus and NUSEX), tend to drag the fit towards small neutrino mixings and to stabilize it at intermediate values. Of course, the overall compatibility of the different data sets might be no longer guaranteed if the total uncertainties were smaller. Therefore, it is very important that the magnitude of the experimental systematic errors in the muon flux distribution (see Sec. II B) is clarified in the running underground experiments (MACRO, Baksan, and SuperKamiokande).

\section{SUMMARY AND CONCLUSIONS}

We have performed an updated analysis of the available upward-going muon data (which disfavor neutrino oscillations), and a comparison with the atmospheric sub-GeV and multi$\mathrm{GeV}$ data (which globally favor neutrino oscillations). We have worked out the oscillation constraints placed by the observed upward-going muon zenithal distribution, and identified the zones of the two- and three-flavor mass-mixing parameter space where all the atmospheric data can be reconciled. In general, the $\nu_{\mu} \leftrightarrow \nu_{e}$ oscillation channel provides a better

fit than the $\nu_{\mu} \leftrightarrow \nu_{\tau}$ channel, thus privileging searches with long-baseline reactors. Large neutrino mixing, including threefold maximal mixing, is generally disfavored. The "minimum sacrifice" fit of Refs. 47,48, that discards, in part, the atmospheric multi-GeV data, is not significantly altered by the inclusion of the upward-going muon information. 


\section{ACKNOWLEDGMENTS}

We acknowledge fruitful discussions with T. Montaruli. We thank S. Mikheyev and Y. Totsuka for useful correspondence. The work of A.M. was supported in part by Ministero dell'Università e della Ricerca Scientifica and in part by INFN. 


\section{TABLES}

TABLE I. Detector characteristics, experimental data, and theoretical expectations for upward-going muons. Theoretical expectations refer to our calculations using Bartol neutrino fluxes [36], MRS(G) structure functions [37]38], and Lohmann-Kopp-Voss muon energy losses in standard rock [40]. See the text for details and comments.

\begin{tabular}{|c|c|c|c|c|c|c|c|c|c|c|}
\hline Experiment & \multicolumn{2}{|c|}{ Kamiokande 19} & \multicolumn{2}{|c|}{ Baksan 22,21} & \multicolumn{2}{|c|}{ MACRO [24] } & \multicolumn{2}{|c|}{ IMB-1,2 25. ${ }^{\mathrm{d}}$} & \multicolumn{2}{|c|}{ SuperKamioka 28] } \\
\hline Depth (m.w.e.) & \multicolumn{2}{|l|}{2700} & \multicolumn{2}{|c|}{850} & \multicolumn{2}{|l|}{3700} & \multicolumn{2}{|c|}{1570} & \multicolumn{2}{|l|}{2700} \\
\hline Live time (yr) & \multicolumn{2}{|l|}{$\sim 7$} & \multicolumn{2}{|l|}{11.94} & \multicolumn{2}{|l|}{3.06} & \multicolumn{2}{|l|}{2.53} & \multicolumn{2}{|l|}{0.63} \\
\hline Technique & \multicolumn{2}{|c|}{ Cherenkov } & \multicolumn{2}{|c|}{ scintillator } & \multicolumn{2}{|c|}{ scint.+tracking } & \multicolumn{2}{|c|}{ Cherenkov } & \multicolumn{2}{|c|}{ Cherenkov } \\
\hline Dimensions $\left(\mathrm{m}^{3}\right)$ & \multicolumn{2}{|c|}{$\pi 7.8^{2} \times 16.1$} & \multicolumn{2}{|c|}{$17 \times 17 \times 11$} & \multicolumn{2}{|c|}{$12 \times 77 \times 9$} & \multicolumn{2}{|c|}{$18 \times 17 \times 22.5$} & \multicolumn{2}{|c|}{$\pi 16.9^{2} \times 36.2$} \\
\hline Threshold & \multicolumn{2}{|c|}{$E_{\mu}>3 \mathrm{GeV}$} & \multicolumn{2}{|c|}{$E_{\mu}>1 \mathrm{GeV}$} & \multicolumn{2}{|c|}{$E_{\mu}>1 \mathrm{GeV}$} & \multicolumn{2}{|c|}{$E_{\mu} \gtrsim 1.8 \mathrm{GeV}$} & \multicolumn{2}{|c|}{$E_{\mu} \gtrsim 6 \mathrm{GeV}$} \\
\hline No. of observed $\mu$ 's & \multicolumn{2}{|c|}{364} & \multicolumn{2}{|c|}{558} & \multicolumn{2}{|c|}{255} & \multicolumn{2}{|c|}{430} & 267 & \\
\hline $\cos \theta$ & muon flux & & muon flu & & muon flux & & muon flux & & muon flux & \\
\hline bin & expt. $\pm \sigma_{\text {stat }}$ & theo. & expt. $\pm \sigma_{\text {stat }}$ & theo. & expt. $\pm \sigma_{\text {stat }}$ & theo. & expt. $\pm \sigma_{\text {stat }}$ & theo. & expt. $\pm \sigma_{\text {stat }}$ & theo. \\
\hline$[-1,-0.9]$ & $1.44 \pm 0.27$ & 1.54 & $2.01 \pm 0.22$ & 2.04 & $1.10 \pm 0.22$ & 2.04 & $1.72 \pm 0.29$ & 1.77 & $0.86 \pm 0.25$ & 1.24 \\
\hline$[-0.9,-0.8]$ & $0.89 \pm 0.20$ & 1.62 & $2.54 \pm 0.27$ & 2.13 & $1.03 \pm 0.23$ & 2.13 & $1.03 \pm 0.23$ & 1.85 & $1.35 \pm 0.31$ & 1.30 \\
\hline$[-0.8,-0.7]$ & $1.54 \pm 0.28$ & 1.70 & $2.73 \pm 0.27$ & 2.24 & $2.22 \pm 0.35$ & 2.24 & $1.78 \pm 0.30$ & 1.95 & $0.98 \pm 0.25$ & 1.37 \\
\hline$[-0.7,-0.6]$ & $1.44 \pm 0.27$ & 1.81 & $2.43 \pm 0.30$ & 2.37 & $3.23 \pm 0.46$ & 2.37 & $1.49 \pm 0.28$ & 2.07 & $1.78 \pm 0.34$ & 1.47 \\
\hline$[-0.6,-0.5]$ & $1.25 \pm 0.25$ & 1.94 & $1.82 \pm 0.30$ & 2.53 & $1.54 \pm 0.34$ & 2.53 & $1.97 \pm 0.30$ & 2.21 & $1.26 \pm 0.28$ & 1.57 \\
\hline$[-0.5,-0.4]$ & $1.41 \pm 0.27$ & 2.11 & $2.70 \pm 0.34$ & 2.73 & $2.68 \pm 0.51$ & 2.73 & $2.16 \pm 0.33$ & 2.40 & $1.63 \pm 0.31$ & 1.72 \\
\hline$[-0.4,-0.3]$ & $2.24 \pm 0.35$ & 2.33 & $2.16 \pm 0.34$ & 3.00 & $3.12 \pm 0.64$ & 3.00 & $2.60 \pm 0.36$ & 2.65 & $1.32 \pm 0.29$ & 1.91 \\
\hline$[-0.3,-0.2]$ & $2.69 \pm 0.38$ & 2.59 & $4.60 \pm 0.57$ & 3.31 & $4.57 \pm 0.94$ & 3.31 & $2.86 \pm 3.93$ & 2.93 & $1.66 \pm 0.31$ & 2.13 \\
\hline$[-0.2,-0.1]$ & $3.94 \pm 0.46$ & 2.93 & $5.50 \pm 0.80$ & 3.72 & $4.01 \pm 1.21$ & 3.72 & $3.93 \pm 0.49$ & 3.30 & $2.71 \pm 0.43$ & 2.43 \\
\hline$[-0.1,0]$ & $2.27 \pm 0.33$ & 3.52 & $6.00 \pm 1.37$ & 4.39 & $12.7 \pm 5.18$ & 4.39 & $2.71 \pm 0.49$ & 3.93 & $4.06 \pm 0.52$ & 2.93 \\
\hline Total muon flux ${ }^{\mathrm{a}}$ & $1.91 \pm 0.10^{\mathrm{b}}$ & 2.21 & $3.25 \pm 0.19^{\mathrm{c}}$ & 2.85 & $3.62 \pm 0.55^{\mathrm{c}}$ & 2.85 & $2.22 \pm 0.11^{\mathrm{b}}$ & 2.51 & $1.76 \pm 0.10^{\mathrm{b}}$ & 1.81 \\
\hline
\end{tabular}

${ }^{\mathrm{a}}$ Units: $10^{-13} \mathrm{~cm}^{-2} \mathrm{~s}^{-1} \mathrm{sr}^{-1}$. Total $\mu$ flux (bottom row) $=$ sum of partial $\mu$ fluxes in each $\cos \theta$-bin times the bin width.

${ }^{\mathrm{b}}$ Experimental systematic errors not reported in 19,25, 26, 28,

${ }^{\mathrm{c}}$ Experimental systematic error of the total flux estimated to be $8 \%$ 21,24.

${ }^{\mathrm{d}}$ IMB-1,2,3 26] (3.6 yr live time) did not publish the muon flux distribution. The 617 observed muons were instead divided in "stopping" (85 events, $1.4 \lesssim E_{\mu} \lesssim 2.5 \mathrm{GeV}$ ) and "passing" (532 events, $E_{\mu} \gtrsim 2.5 \mathrm{GeV}$ ). The total muon flux reported in 26 is $2.22 \pm 0.11 \times 10^{-13} \mathrm{~cm}^{-2} \mathrm{~s}^{-1} \mathrm{sr}^{-1}$ (stat. error only). Our theoretical estimates: 102 (stopping) +645 (passing) muons; total muon flux $=2.51 \times 10^{-13} \mathrm{~cm}^{-2} \mathrm{~s}^{-1} \mathrm{sr}^{-1}$. 


\section{REFERENCES}

[1] M. A. Markov and I. M. Zheleznykh, Nucl. Phys. 27, 385 (1961); G. T. Zatsepin and V. A. Kuz'min, Zh. Eksp. Teor. Fiz. 41, 1818 (1961) [Sov. Phys. JETP 14, 1294 (1962)]; see also: K. Greisen, Ann. Rev. Nucl. Science 10, 63 (1960).

[2] T. D. Lee, H. Robinson, M. Schwartz, and R. Cool, Phys. Rev. 132, 1297 (1963).

[3] J. L. Osborne et al., Proc. Phys. Soc. London 86, 93 (1965).

[4] R. Cowsik et al., Proc. Indian Acad. Sci. A 63, 217 (1966).

[5] Johannesburg Mine experiment, F. Reines et al., Phys. Rev. Lett. 15, 429 (1965); M. F. Crouch et al., Phys. Rev. D 18, 2239 (1978).

[6] Kolar Gold Field experiment, C. Achar et al., Phys. Lett. 18, 196 (1965); M. R. Krishaswamy et al., Proc. R. Soc. London A 323, 489 (1971).

[7] K. V. L. Sarma and L. Wolfenstein, Phys. Lett. B 61, 77 (1976).

[8] V. Barger, K. Whisnant, D. Cline, and R. J. N. Phillips, Phys. Lett. B 93, 194 (1980); V. Barger, K. Whisnant, and R. J. N. Phillips, Phys. Rev. D 22, 1636 (1980).

[9] T. K. Gaisser, F. Halzen, and T. Stanev, Phys. Rep. 258, 173 (1995); 271, 355(E) (1996).

[10] T. K. Gaisser, in Neutrino '96, Proceedings of the 17th Conference on Neutrino Physics and Astrophysics, Helsinki, 1996, to appear.

[11] T. Stanev, in TAUP '95, Proceedings of the 4th International Workshop on Theoretical and Phenomenological Aspects of Underground Physics, Toledo, Spain, edited by A. Morales, J. Morales, and J. A. Villar [Nucl. Phys. B (Proc. Suppl.) 48, 165 (1996)].

[12] M. Honda, T. Kajita, K. Kasahara, and S. Midorikawa, Phys. Rev. D 52, 4985 (1995); Prog. Theor. Phys. Suppl. 123, 483 (1996). 
[13] W. Frati, T. K. Gaisser, A. K. Mann, and T. Stanev, Phys. Rev. D 48, 1140 (1993).

[14] E. Akhmedov, P. Lipari, and M. Lusignoli, Phys. Lett. B 300, 128 (1993).

[15] P. Lipari, M. Lusignoli, and F. Sartogo, Phys. Rev. Lett. B 74, 4384 (1995).

[16] Kamiokande Collaboration, Y. Oyama et al., Phys. Rev. D 39, 1481 (1989).

[17] Kamiokande Collaboration, M. Mori et al., Phys. Lett. B 270, 89 (1991).

[18] Kamiokande Collaboration, M. Mori et al., Phys. Rev. D 48, 5505 (1993).

[19] Kamiokande Collaboration, A. Suzuki et al. in the Proceedings of 7th International Workshop on Neutrino Telescopes, Venice, 1996, edited by M. Baldo Ceolin (University of Padova, Italy, 1996) p. 263.

[20] M. M. Boliev, A. V. Butkevich, A. E. Chudakov, S. P. Mikheyev, O. V. Suvorova, and V. N. Zakidyshev, in ICRC '95, Proceedings of the 24th International Cosmic Ray Conference, Rome (Arti Grafiche Editoriali, Urbino, Italy, 1995) Vol 1, p. 686; ibidem, p. 722 .

[21] S. Mikheyev, in the Proceedings of the 8th Rencontres de Blois on Neutrinos, Dark Matter, and the Universe, Blois, France, 1996, to appear.

[22] M. M. Boliev, E. V. Bugaev, A. V. Butkevich, A. E. Chudakov, S. P. Mikheyev, O. V. Suvorova, and V. N. Zakidyshev, in DARK '96, Proceedings of the International Workshop on Aspects of Dark Matter in Astrophysics and Particle Physics, Heidelberg, Germany, 1996, to appear.

[23] MACRO Collaboration, S. Ahlen et al., Phys. Lett. B 357, 481 (1995).

[24] MACRO Collaboration, F. Ronga et al., in Neutrino '96 [10], to appear.

[25] D. W. Casper, in the Proceedings of 3rd International Workshop on Neutrino Telescopes, Venice, 1991, edited by M. Baldo Ceolin (University of Padova, Italy, 1991) p. 213. 
[26] IMB Collaboration, R. Becker-Szendy et al., Phys. Rev. Lett. 69, 1010 (1992).

[27] SuperKamiokande Collaboration, K. Young et al., in the Joint APS/AAPT 1997 Meeting, Washington DC, April 1997, to appear in the Proceedings; also available at the URL hhp://www.phys.washington.edu/ young/superk/drafts/aps97.html .

[28] SuperKamiokande Collaboration, to be presented by J. G. Learned in ICRC' '97, 25th International Cosmic Rays Conference, Durban, South Africa, 1997 astro-ph/9705197).

[29] G. L. Fogli, E. Lisi, and D. Montanino, Phys. Rev. D 49, 3626 (1994); Astropart. Phys. 4, 177 (1995); Phys. Rev. D 96, 2048 (1996).

[30] G. L. Fogli and E. Lisi, Phys. Rev. D 52, 2775 (1995).

[31] G. L. Fogli, E. Lisi, D. Montanino, and G. Scioscia, Phys. Rev. D55, 4385 (1997).

[32] G. L. Fogli, E. Lisi, and G. Scioscia, Phys. Rev. D 52, 5334 (1995); Bari University Report No. BARI-TH/259-97, hep-ph/9702298, to appear in Phys. Rev. D.

[33] NUSEX Collaboration, M. Aglietta et al., Europhys. Lett. 15, 559 (1991).

[34] T. K. Gaisser and T. Stanev, Phys. Rev. D 30, 985 (1984).

[35] G. Auriemma, M. Felcini, P. Lipari, and J. L. Stone, Phys. Rev. D 37, 665 (1988).

[36] V. Agrawal, T. K. Gaisser, P. Lipari, and T. Stanev, Phys. Rev. D 53 1314, (1996).

[37] A. D. Martin, W. J. Stirling, and R. G. Roberts, Int. J. Mod. Phys. A 10, 2885 (1995); Phys. Lett. B 354, 155 (1995); Phys. Rev. D 51, 4756, (1995).

[38] H. Plotow-Besch, "PDFLIB: The Parton Density Function Library," User's Manual, Version 7.07, available at the URL http://consult.cern.ch/writeup/pdflib.

[39] H. Plotow-Besch, Int. J. Mod. Phys. A 10, 2901 (1995).

[40] W. Lohmann, R. Kopp, and R. Voss, CERN Yellow Report No. 85-03 (unpublished). 
[41] L. V. Volkova, Yad. Fiz. 31, 1510 (1980) [Sov. J. Nucl. Phys. 31, 784 (1980)].

[42] E. Eichten, I. Hinchliffe, K. Lane, and C. Quigg, Rev. Mod. Phys. 56, 579 (1984); 58, 1065(E) (1986).

[43] J. Morfin and W. K. Tung, Z. Phys. C 52, 13 (1991).

[44] L. B. Bezrukov and E. V. Bugaev, in ICRC '81, Proceedings of the 7th International Cosmic Ray Conference, Paris (Centre d'Etudes Nucleaires de Saclay, Gif-sur-Yvette, France, 1981), Vol. 7, p. 102.

[45] Review of Particle Physics, R. M. Barnett et al., Phys. Rev. D 54, 1 (1996).

[46] Kamiokande Collaboration, Y. Fukuda et al., Phys. Lett. B 335, 237 (1994).

[47] C. Y. Cardall and G. M. Fuller, Phys. Rev. D 53, 4421 (1996).

[48] G. L. Fogli, E. Lisi, D. Montanino, and G. Scioscia, University of Bari Report No. BARI-TH/273-97, hep-ph/9706230, to appear in Phys. Rev. D.

[49] P. F. Harrison, D. H. Perkins, and W. G. Scott, Phys. Lett. B 349, 137 (1995); 396, 186 (1997).

[50] C. Giunti, C. W. Kim, and J. D. Kim, Phys. Lett. B 352, 357 (1995). 


\section{FIGURES}

FIG. 1. Compilation of upward-going muon data and expectations for different experiments: Kamiokande [19], Baksan [21,22], MACRO [24], SuperKamiokande [28] IMB-1,2 [25], and IMB-1,2,3 [26]. Dots with error bars: Experimental data with $1 \sigma$ statistical uncertainties. Solid histograms:

our calculations. Dashed histograms: Experimental simulations. $\theta$ is the zenith angle. See also Table I.

FIG. 2. Comparison of all upward-going muon spectra, rescaled to a common threshold of 1 GeV. (a) Separated data. (b) Combined data (as used in our analysis). See the text for details.

FIG. 3. Results of the oscillation fit in the two-flavor oscillation channel $\nu_{\mu} \leftrightarrow \nu_{\tau}$, shown as C.L. contours in the $\left(\tan ^{2} \psi, m^{2}\right)$ plane. The allowed regions are marked by stars.

FIG. 4. Results of the oscillation fit in the two-flavor oscillation channel $\nu_{\mu} \leftrightarrow \nu_{e}$, shown as C.L. contours in the $\left(\tan ^{2} \phi, m^{2}\right)$ plane. The allowed regions are marked by stars.

FIG. 5. Bounds placed by all upward-going muon data in the three-flavor oscillation space $\left(m^{2}, \tan ^{2} \phi, \tan ^{2} \psi\right)$. The allowed regions are marked by stars.

FIG. 6. As in Fig. 5, but with the addition of the atmospheric sub-GeV (S) and multi-GeV binned $(\mathrm{Mb})$ data (analyzed as in [31]). 

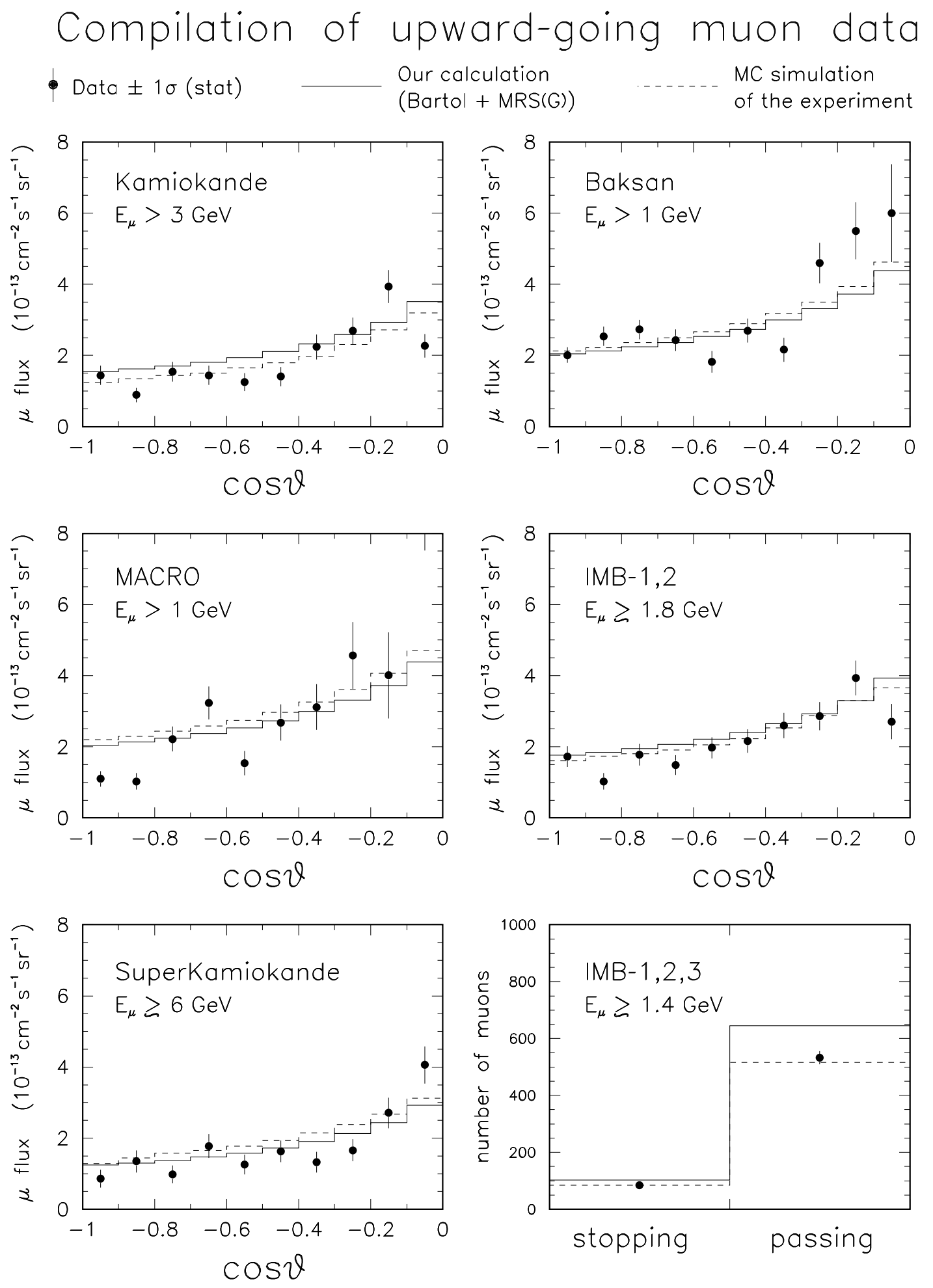

FIG. 1. Compilation of upward-going muon data and expectations for different experiments: Kamiokande [19], Baksan [21,22], MACRO [24], SuperKamiokande [28] IMB-1,2 [25], and IMB-1,2,3 [26]. Dots with error bars: Experimental data with $1 \sigma$ statistical uncertainties. Solid histograms: our calculations. Dashed histograms: Experimental simulations. $\theta$ is the zenith angle. See also Table I. 
Comparison of upward-going muon data
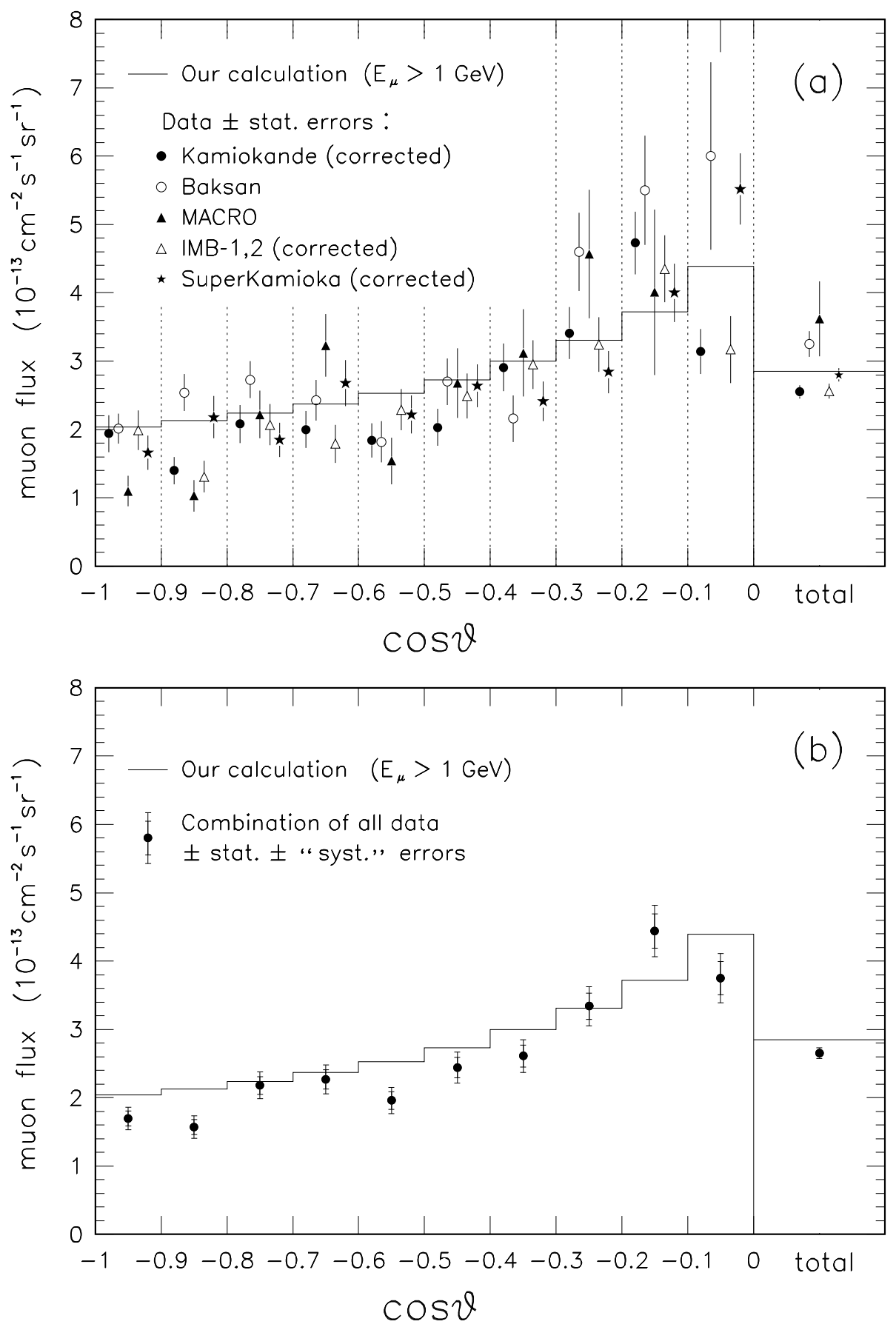

FIG. 2. Comparison of all upward-going muon spectra, rescaled to a common threshold of 1 GeV. (a) Separated data. (b) Combined data (as used in our analysis). See the text for details. 
$\nu_{\mu} \leftrightarrow \nu_{\tau}$ oscillations
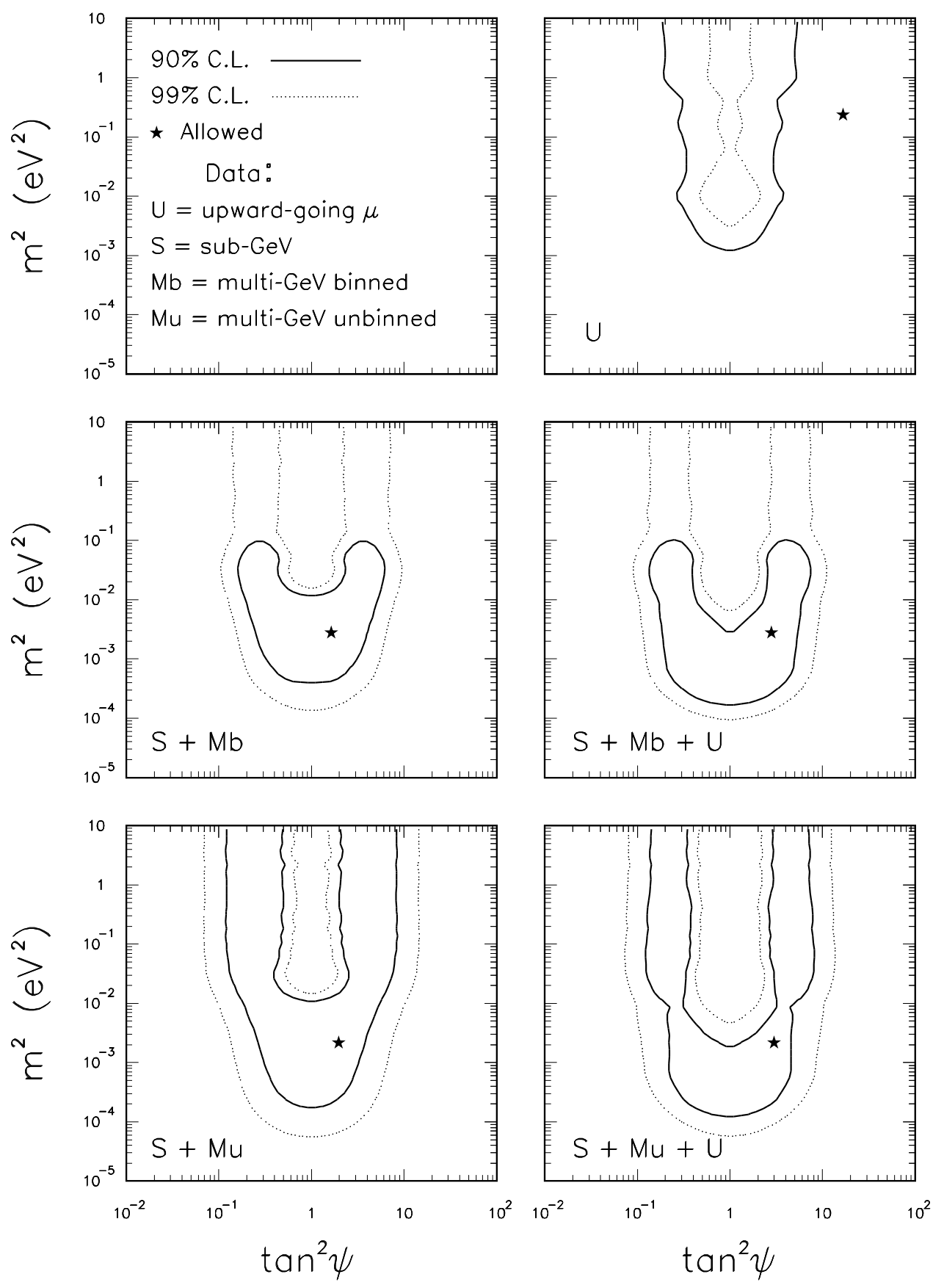

FIG. 3. Results of the oscillation fit in the two-flavor oscillation channel $\nu_{\mu} \leftrightarrow \nu_{\tau}$, shown as C.L. contours in the $\left(\tan ^{2} \psi, m^{2}\right)$ plane. The allowed regions are marked by stars. 


\section{$\nu_{\mu} \leftrightarrow \nu_{\mathrm{e}}$ oscillations}
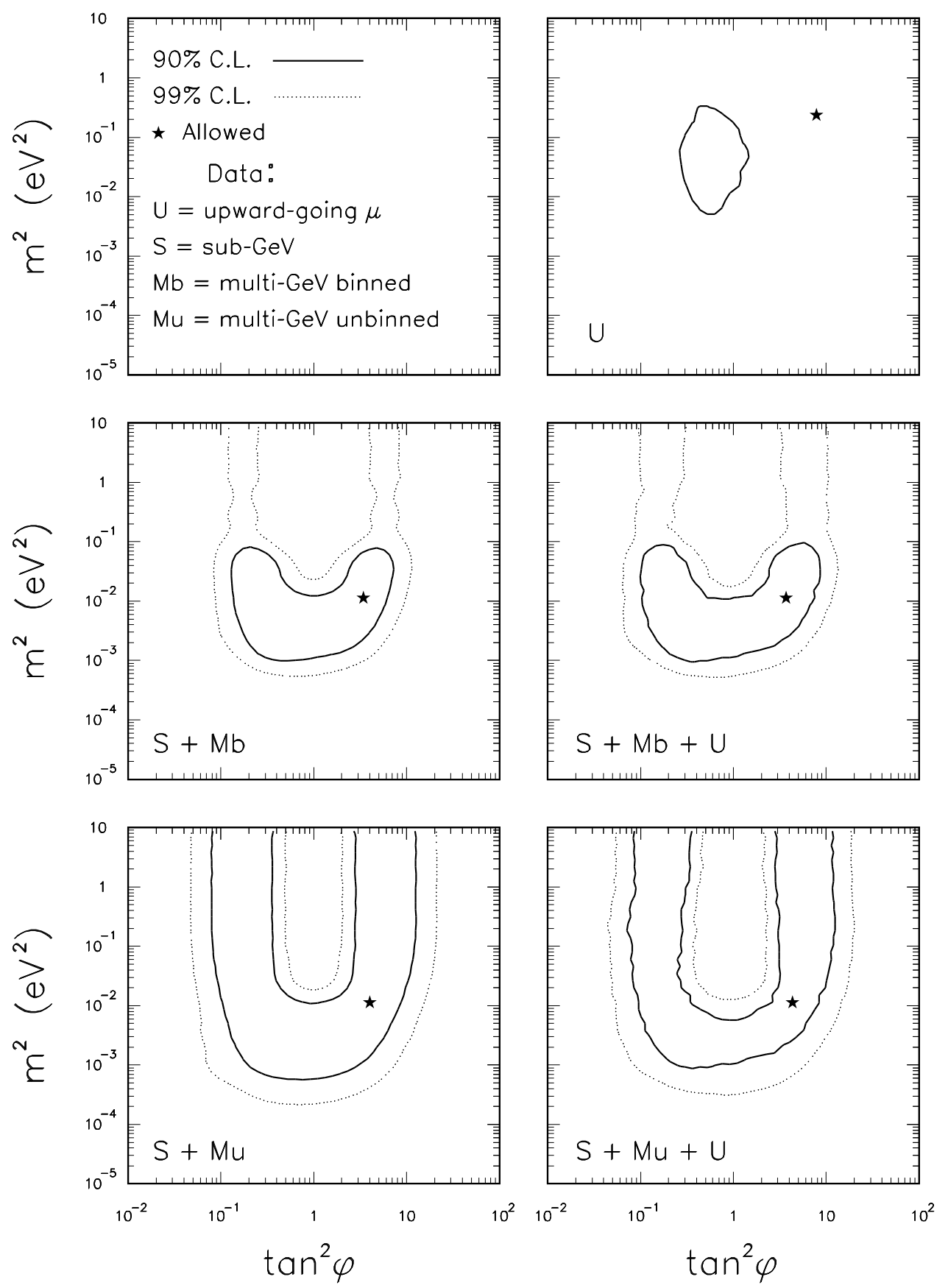

FIG. 4. Results of the oscillation fit in the two-flavor oscillation channel $\nu_{\mu} \leftrightarrow \nu_{e}$, shown as C.L. contours in the $\left(\tan ^{2} \phi, m^{2}\right)$ plane. The allowed regions are marked by stars. 


\section{$3 \nu$ oscillations, upward-going $\mu$}
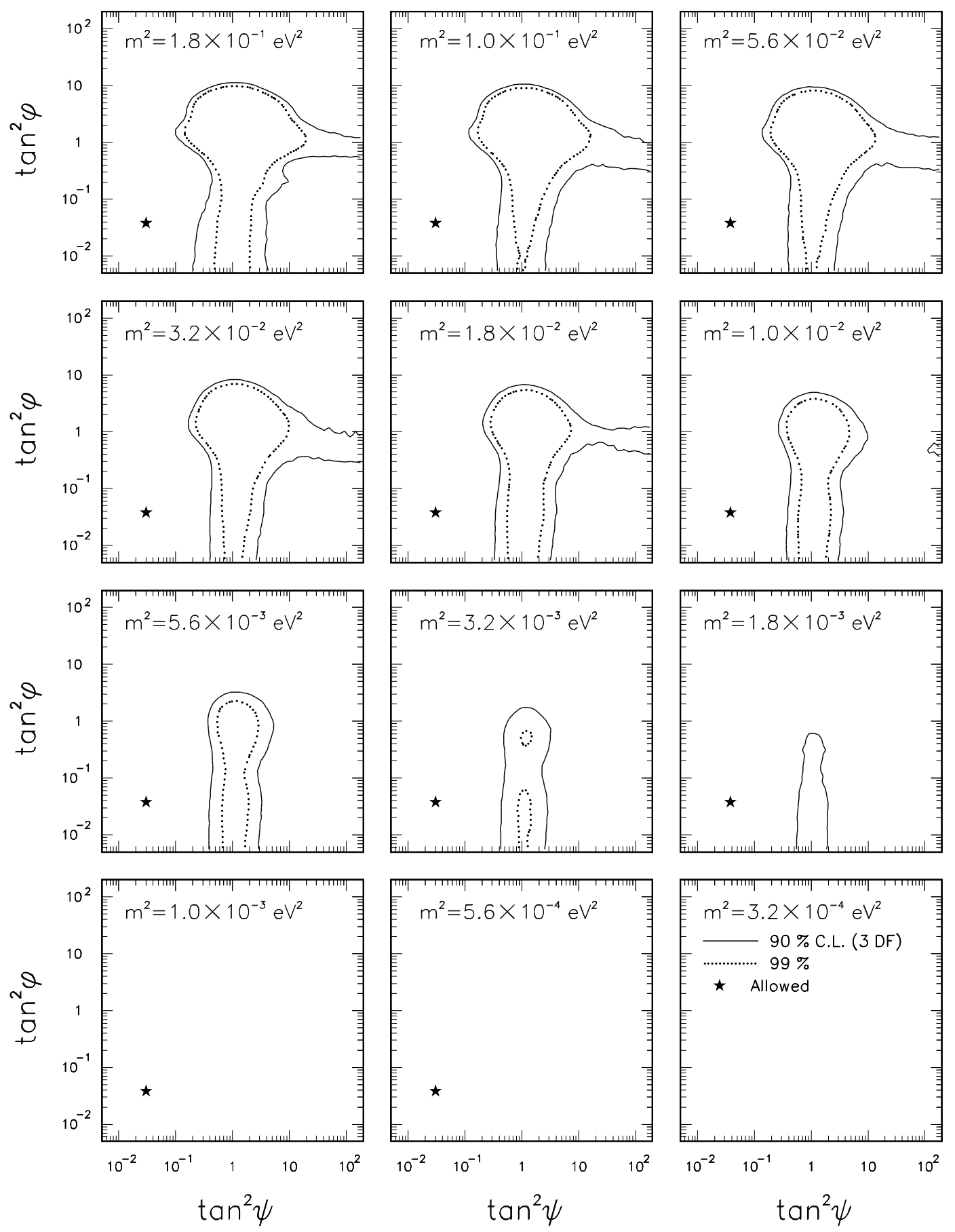

FIG. 5. Bounds placed by all upward-going muon data in the three-flavor oscillation space $\left(m^{2}, \tan ^{2} \phi, \tan ^{2} \psi\right)$. The allowed regions are marked by stars. 
$3 \nu$ oscillations, $U+S+M b$ data
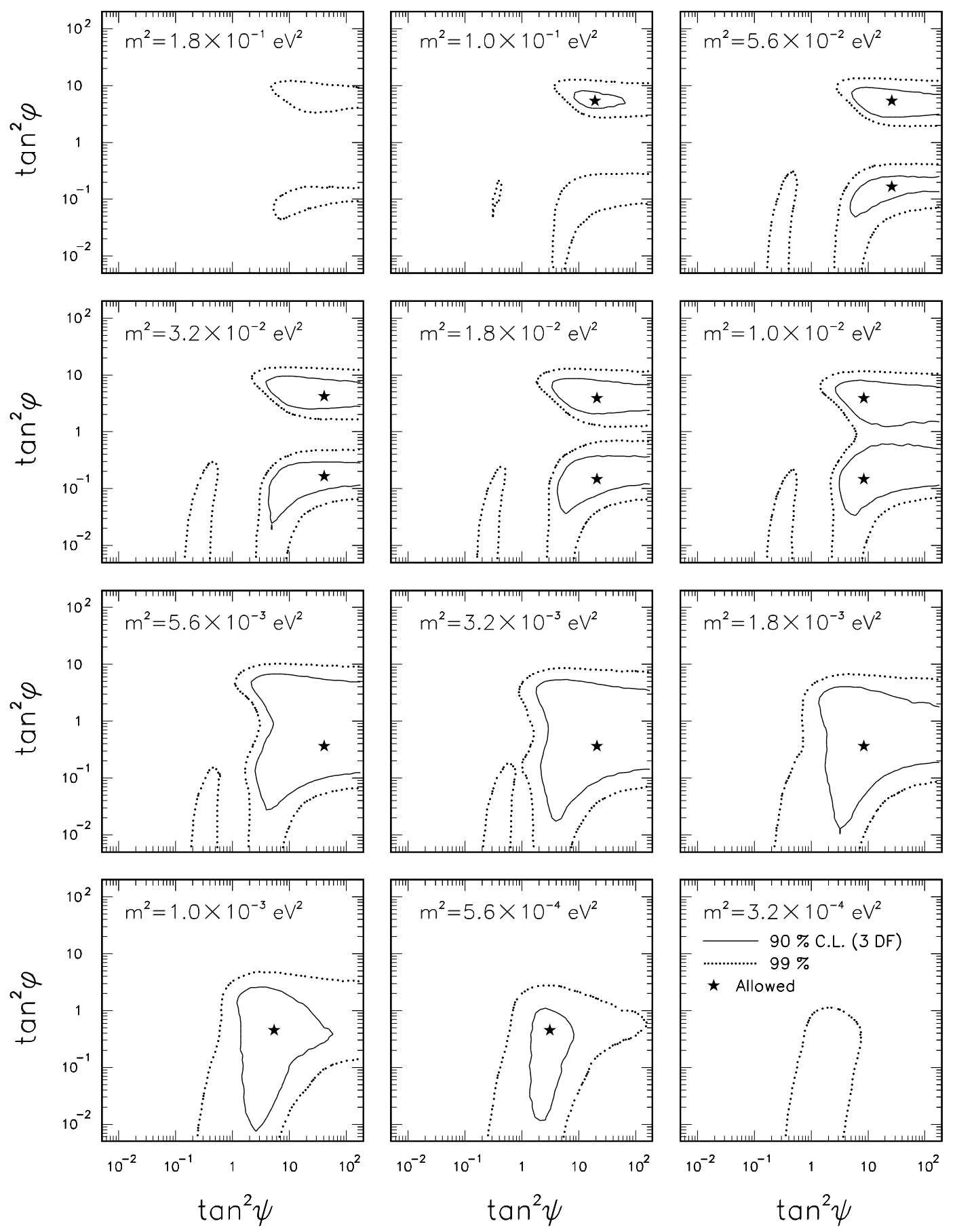

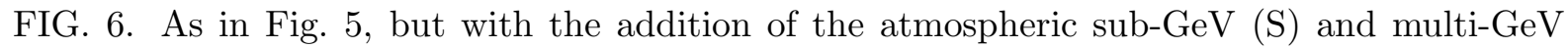
binned $(\mathrm{Mb})$ data (analyzed as in 31]). 